\title{
Episodic ejection from super-massive black holes
}

\author{
Lakshmi Saripalli, ${ }^{1} \dagger$ Ravi Subrahmanyan ${ }^{1}$ and Richard W. Hunstead ${ }^{2}$ \\ ${ }^{1}$ Raman Research Institute, Bangalore, India \\ ${ }^{2}$ School of Physics, University of Sydney, Australia \\ email: lsaripal@rri.res.in, rsubrahm@rri.res.in,rwh@physics.usyd.edu.au
}

\begin{abstract}
Episodic activity in super-massive black holes is shown by radio galaxies exhibiting 'double-double' radio morphologies (Subrahmanyan et al. 1996, Schoenmakers et al. 2000). Spectacular examples showing a renewal of beam activity in the form of new beams emerging within relic radio lobes of previous activity have placed the phenomenon of recurrence in AGN outflows on a firm footing.

By using the SUMSS and WENSS GRG samples, we infer that on timescales of order a few million years, low luminosity radio sources are more likely to exhibit episodic behaviour in the accretion on to their supermassive black holes as compared to the more powerful radio galaxies.
\end{abstract}

Keywords. Galaxies: active - galaxies: nuclei - accretion

\section{Introduction}

Extended radio galaxies provide a historical record of the central activity: the extended radio lobes were built up over the source lifetime and represent a history of the beam power from the supermassive black hole at the centre of the galaxy. Radio galaxies of the largest linear sizes, the giant radio galaxies (GRGs; linear size $>0.7 \mathrm{Mpc}$ ) are particularly suitable for such studies. Their large ages imply that there is a higher probability that they were witness to changes in activity at the centres during their lifetime. Additionally, abrupt changes in the activity of the central engine would take longer times to propagate over the giant radio lobes, thus increasing the likelihood of observing such interruptions and restarting in these sources.

\section{Recurrence in nuclear activity in GRGs}

There are four samples of GRGs available today that form a resource from which compilations of re-starting radio galaxies may be made. They are the WENSS $325 \mathrm{MHz}$ sample (Schoenmakers et al. 2001), two NVSS 1.4 GHz samples that have been formed using different angular size criteria (Lara et al. 2001 and Machalski et al. 2001) and the SUMSS $843 \mathrm{MHz}$ sample (Saripalli et al. 2005).

In this work we use two samples of GRGs: the WENSS and the SUMSS samples, to explore the phenomenon of episodic ejection from supermassive black holes. WENSS (47 GRGs over 8100 sq. deg.) and SUMSS (18 GRGs over 2100 sq. deg.) have complete redshift information and are matched in their selection criteria. Given that these samples have been made using a lower limit of $5^{\prime}$ for the angular extent, and a lower limit of $0.7 \mathrm{Mpc}$ for the linear size, the two samples are expected to contain all giants with redshift $z \leqslant 0.13$ that have radio luminosities above the survey sensitivity limits.

$\dagger$ Present address: Raman Research Institute, C. V. Raman Avenue, Sadashivanagar, Bangalore 560 080, India. 


\section{The sample of low redshift restarting GRGs}

The SUMSS GRG sample has four radio galaxies with $z \leqslant 0.13$ and in the WENSS sample there are 25 such sources. Together, there are $29 z \leqslant 0.13$ GRGs, and their radio powers have a clear bimodal distribution. 19 GRGs form a distinct population at lower radio power with a median $\sim 2 \times 10^{24} \mathrm{~W} \mathrm{~Hz}^{-1}$; the remaining 10 GRGs have relatively higher power with a median $\sim 2 \times 10^{25} \mathrm{~W} \mathrm{~Hz}^{-1}$.

We have used the following morphological indicators for recognizing a restarting of activity: (i) Twin pairs of nested lobes or sources with 'double-double' morphology, or alternately (ii) a pair of outer lobes together with inner emission features that are observed to terminate at hot/warm spots well recessed from the ends of the lobes. Among the 10 powerful GRGs as many as 9 are in the ON state (currently active) of which only 1 may be classified as restarting. There is only one source among the 10 that appears to be in the $\mathrm{OFF}$ state, i.e. in a relic phase with neither hot-spots at the lobe ends nor inner double structure. In contrast, among the low power GRGs we find that 15 of the 19 sources are in the ON state; however, of the 15 as many as 7 appear to be in the restarting phase. Additionally, 4 of the 19 low power GRGs appear to be in the relic phase and show no signs of jets or hot-spots.

\section{Discussion}

The fraction of visible relics is small. This must imply that the time for which these sources remain visible after the central beams switch OFF is small as compared to their active lifetimes. We infer that that the low power GRG source population is more vulnerable to restarting, since a significantly greater fraction of low power sources are observed to be in the restarting phase as compared to the higher power source population. The low power GRGs, with radio power below the FR-I/II dividing line, are inferred to have relatively short duty cycles in their central activity, alternating between ON and OFF states. In contrast, in the high power GRGs, with radio powers above the FR-I/II dividing line, restarting on timescales of a few Myr is rare.

If giant radio sources, in which recurrence is more easily recognized, are the tail end of the linear size distribution in the radio source population and are not a distinct peculiar population, then our results imply that low and high power radio sources may have very different recurrence rates in their AGN activity: low luminosity sources may have more frequent episodes of accretion on to their supermassive black holes (Marchesini et al. 2004) with timescales of a few Myr.

\section{References}

Lara, L., Cotton, W. D., Feretti, L., Giovannini, G., Marcaide, J. M., Marquez, I. \& Venturi, T. 2001, A\&A, 370, 409

Machalski, J., Jamrozy, M. \& Zola, S. 2001, A\&A, 371, 445

Marchesini, D., Celotti, A. \& Ferrarese, L. 2004, MNRAS, 351, 733

Saripalli, L., Hunstead, R. W., Subrahmanyan, R. \& Boyce, E. 2005, AJ, 130, 896

Schoenmakers, A. P., de Bruyn, A. G., Roettgering, H. J. A. \& van der Laan, H. 2001, A\&A, 374,861

Schoenmakers, A. P., de Bruyn, A. G., Roettgering, H. J. A., van der Laan, H. \& Kaiser, C. R. 2000, MNRAS, 315, 371

Subrahmanyan, R., Saripalli, L. \& Hunstead, R. W. 1996, MNRAS, 279, 257 